\title{
INCORPORACIÓN DE LAS TIC CON METODOLOGIA B-LEARNING EN LA ENSENAANZA DE PROCESOS INDUSTRIALES
}

\section{Incorporating ICTs with b-learning methodology in teaching industrial processes}

Incorporação das TIC com metodologia b-learning no ensino de processos industriais

\section{RECIBIDO: 18 DE SEPTIEMBRE DE 2012}

Karolina González Guerrero (Colombia) Universidad Militar Nueva Granada

karolina.gonzalez@unimilitar.edu.co

Licenciada en Electrónica de la Universidad Pedagógica Nacional, magíster en Educación de la Pontificia Universidad Javeriana, directora del Centro de Investigaciones e investigadora del grupo PYDES de la

Universidad Militar Nueva Granada.

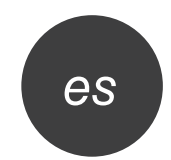

\section{RESUMEN}

Se presenta un estudio de caso descriptivo de corte cualitativo que busca evaluar la implementación y el uso de las tecnologías de la información y la comunicación (TIC) basándose en la metodología b-learning en los procesos pedagógicos del programa de Ingeniería Industrial en la Universidad Distrital Francisco José de Caldas. Se recurre a la utilización de registros descriptivos, observación participante y entrevistas no estructuradas para analizar y comparar el rendimiento académico de los estudiantes en un curso donde se implementa b-learning y el de aquellos en un curso con metodología tradicional. Se espera que este trabajo estimule la participación e integración de los procesos de comunicación entre los actores del proceso educativo y por lo tanto se generen habilidades de aprendizaje autónomo y significativo en los estudiantes.

PALABRAS CLAVES: B-learning, entornos virtuales, formación de ingenieros.
EVALUADO: 11 DE FEBRERO DE 2013

ACEPTADO: 18 DE MARZO DE 2013
Alidis Cristina Contreras Bravo (Colombia) Corporación Universitaria del Caribe

Ingeniera civil y especialista en Gerencia de Proyectos de la Universidad de Cartagena; docente de hora cátedra en la Corporación Universitaria del Caribe.

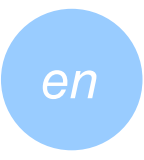

ABSTRACT

We present a descriptive case study of a qualitative kind that seeks to assess the implementation and use of information communication and technology (ICT) based on b-learning methodology in learning processes Industrial Engineering program at the District University Francisco José de Caldas. Descriptive records, participant observation and unstructured interviews are used to analyze and compare the academic performance of students in a course where $b$-learning is implemented and those in a course with traditional methodology. It is expected that this work will stimulate the participation and integration of communication processes between the actors of the educational process and thus generate meaningful and autonomous learning skills in students.

KEY WORDS: B-learning, virtual environments, training of engineers.
Leonardo Emiro Contreras Bravo (Colombia)

Universidad Distrital Francisco José de Caldas

lecontrerasb@udistrital.edu.co.

Ingeniero mecánico de la Universidad Francisco de Paula Santander, magíster en Ingeniería, Materiales y Procesos de la Universidad Nacional de Colombia, docente de planta de la Universidad Distrital Francisco José de Caldas en Bogotá.

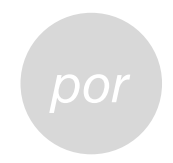

\section{RESUMO}

Apresenta-se um estudo de caso descritivo de corte qualitativo que procura avaliar a implementação e o uso das tecnologias da informação e a comunicação (TIC) baseando-se na metodologia b-learning nos processos pedagógicos do programa de Engenharia Industrial na Universidade Distrital Francisco José de Caldas. Recorre-se à utilização de registros descritivos, observação participante e entrevistas não estruturadas para analisar e comparar o rendimento acadêmico dos estudantes em um curso onde se implementa o b-learning e/ou de aqueles em um curso com metodologia tradicional. Espera-se que este trabalho estimule a participação e integração dos processos de comunicação entre os atores do processo educativo e pelo tanto se gerem habilidades de aprendizado autônomo e significativo nos estudantes.

PALAVRAS CHAVES: B-learning, entornos virtuais, formação de engenheiros.

PARA CITAR este artículo / to CITE this ARTICLE / PARA CITAR ESTE ARTIGO: 

El avance tecnológico y especialmente las tecnologías de la información y la comunicación han influido en muchos campos, uno de estos es el sector educativo (González y León, 2010), en el cual es posible apreciar algunos cambios, como los casos del tablero y el marcador, que han sido reemplazados por los proyectores de multimedia (video beam), las carteleras y los centros de copiado, que fueron reemplazados por el espacio virtual o los correos electrónicos, que posibilitan mantener una comunicación en tiempo real. Por lo tanto, los parámetros culturales actuales se encuentran atravesados y teñidos por estas nuevas tecnologías. En esta dirección, Garrido (2005) afirma que "el mercado del futuro y las demandas laborales girarán en torno a la información y al manejo de la información” y "los medios transforman al mundo y están transformando la enseñanza” (p 246).

La asignatura Procesos Industriales II, de la carrera de Ingeniería Industrial de la Universidad Distrital Francisco José de Caldas empezó a dictarse en el año 1997. A partir del primer semestre del año 2007 se introduce el uso de la plataforma Moodle a la asignatura, pero con uso limitado, puesto que no se hace uso de sus múltiples opciones, como foros, tareas, quices, lecturas evaluativas, entre otras; solo es usado como un repositorio de documentos (herramienta para descargar presentaciones de clase, guías de laboratorio y archivos de lectura complementaria).

Por tal motivo, en el segundo semestre de 2010 se ha introducido como recurso pedagógico adicional y complementario la utilización de un Aula Virtual, que incluye el desarrollo de una serie de actividades a distancia. El diseño está orientado a un sistema de $b$-learning (aprendizaje mixto o mezclado), que combina educación a distancia y semipresencial, ya que la formación de los ingenieros exige la búsqueda permanente de alternativas pedagógicas, así como el continuo aprovechamiento de los recursos tecnológicos que ofrece el mundo actual, para lograr mejores niveles de capacitación (Andrade, 2007).

Este proyecto de innovación tecnológica, bajo una modalidad b-learning, pretende ser un aporte a los nuevos procesos pedagógicos, necesarios en las aulas bajo la metodología virtual y semipresencial, con el propósito de aprovechar las oportunidades de mejora para el aprendizaje de los alumnos y facilitar la búsqueda del mismo, utilizando herramientas tecnológicas de apoyo que estimulen las habilidades del alumno en beneficio de la construcción de conocimientos.

El propósito de este proyecto piloto es iniciar la creación de bases que permitan la inclusión de las tecnologías de la información y la comunicación (TIC) en los programas de las asignaturas correspondientes a Ingeniería Industrial para evaluar ventajas o desventajas de su uso.

\section{Metodología}

La siguiente investigación corresponde a un tipo de investigación cualitativa (Rodríguez, Gil y García, 1999); hace registros descriptivos de los fenómenos que son estudiados mediante técnicas como la observación participante y las entrevistas no estructuradas; es decir, el investigador es un observador de la realidad donde ocurren los eventos.

El tipo de estudio es descriptivo, su fundamento es el análisis de características que buscan especificar las propiedades importantes de personas o grupos; se seleccionan una serie de interrogantes y se mide cada una de ellas independientemente, para así describir lo que se investiga (Sandoval, 2002). El objetivo, bajo este modelo, es investigar la situación planteada como pregunta de investigación; para esto fue necesario recolectar datos en la Universidad Distrital, específicamente en la asignatura de Procesos Mecánicos, para describir cómo el recurso tecnológico fortalece el proceso de enseñanza y a los cursantes de la asignatura mediante la formulación de estrategias de implementación de las TIC y, especialmente, la metodología b-learning.

| Panorama | pp. 11 - 23 | Volumen 7 | Número 13 Julio - diciembre | 2013 


\section{ASPECTOS TECNOLÓGICOS}

El auge de la tecnología y en especial de la metodología b-learning es tal que se está convirtiéndo en una alternativa que facilita la integración de las nuevas tecnologías y culturas con los métodos de enseñanza tradicionales, por lo que permite combinar, en diferentes proporciones, los recursos presenciales y no presenciales, donde el docente pasa de ser de un agente transmisor a ser un mediador de la construcción de nuevos conocimientos(González, Padilla y Arias, 2010). En este sentido, por ejemplo, se pueden combinar clases magistrales con materiales escritos en soporte electrónico, con la utilización de un espacio virtual, con videos (en el caso de procesos de manufactura), con sesiones de estudio independientes, con la participación en foros, el uso de tutoriales de software de ingeniería, exámenes presenciales, prácticas de laboratorio reales o virtuales, etcétera.

Existen varias posibilidades tecnológicas para implementar un b-learning, cada una con sus ventajas y desventajas; García y Lacleta (2006) nos ofrecen un panorama de cómo han ido evolucionando los entornos de e-learning; no obstante, este texto se limita a los recursos utilizados en la experiencia: el Aula Virtual Moodle (AVM); el Moodle (Modular object-oriented dynamic learning environment). Esta aula virtual no implica un mero repositorio de documentos en formato web o exponer contenidos, sino diseñar propuestas pedagógicas que favorezcan la construcción social del conocimiento de manera autónoma, a través de un proceso de traspaso paulatino del control, como lo señala Rogoff (1998).

\section{AsPECTOS DEL TRABAJO ACADÉMICO}

Según el Acuerdo 009 del 12 de septiembre de 2006 del Consejo Académico de la Universidad Distrital, y los lineamientos expuestos por Arias, González y Padilla (2010), los espacios académicos se han considerado para ser entendidos como asignaturas, cátedras y grupos de trabajo; deben implementar un sistema de créditos académicos; además deben implementar unas horas para la formación del estudiante, definidas como horas de trabajo directo (HTD), horas de trabajo cooperativo (HTC) y de trabajo autónomo (HTA), las cuales se resumen en la tabla 1.

\section{TABLA 1. TIPOS DE HORAS DE TRABAJO ACADÉMICO SEGÚN LA UNIVERSIDAD DISTRITAL FRANCISCO JOSE DE CALDAS}

\section{Horas de trabajo directo (HTD)}

Son horas lectivas de clase a las que deben asistir los estudiantes de manera presencial.

\section{Horas de trabajo cooperativo (HTC)}

Son horas lectivas de clase a las que deben asistir los estudiantes según necesidades, metodologías y programación definida por el profesor, con el propósito que al trabajar individualmente o en grupos desarrollen temáticas, adelanten prácticas, resuelvan problemas, resuelvan ejercicios de aplicación, ensayen aplicaciones, etcétera, siempre bajo la supervisión y asesoría del profesor, según metodologías, programación, propósito, temáticas, prácticas, aplicación, supervisión, asesoría.

\section{Horas de trabajo autónomo (HTA)}

Son horas no lectivas donde el estudiante, sin la presencia del profesor, realiza de manera autónoma tareas y demás actividades necesarias para consolidar su aprendizaje en los diferentes espacios académicos que cursa. 
Con el fin de integrar las consideraciones del Consejo Académico de la Universidad Distrital Francisco José de Caldas con los lineamientos de la educación a distancia, se plantea la metodología de trabajo académico que se muestra en la figura 1.
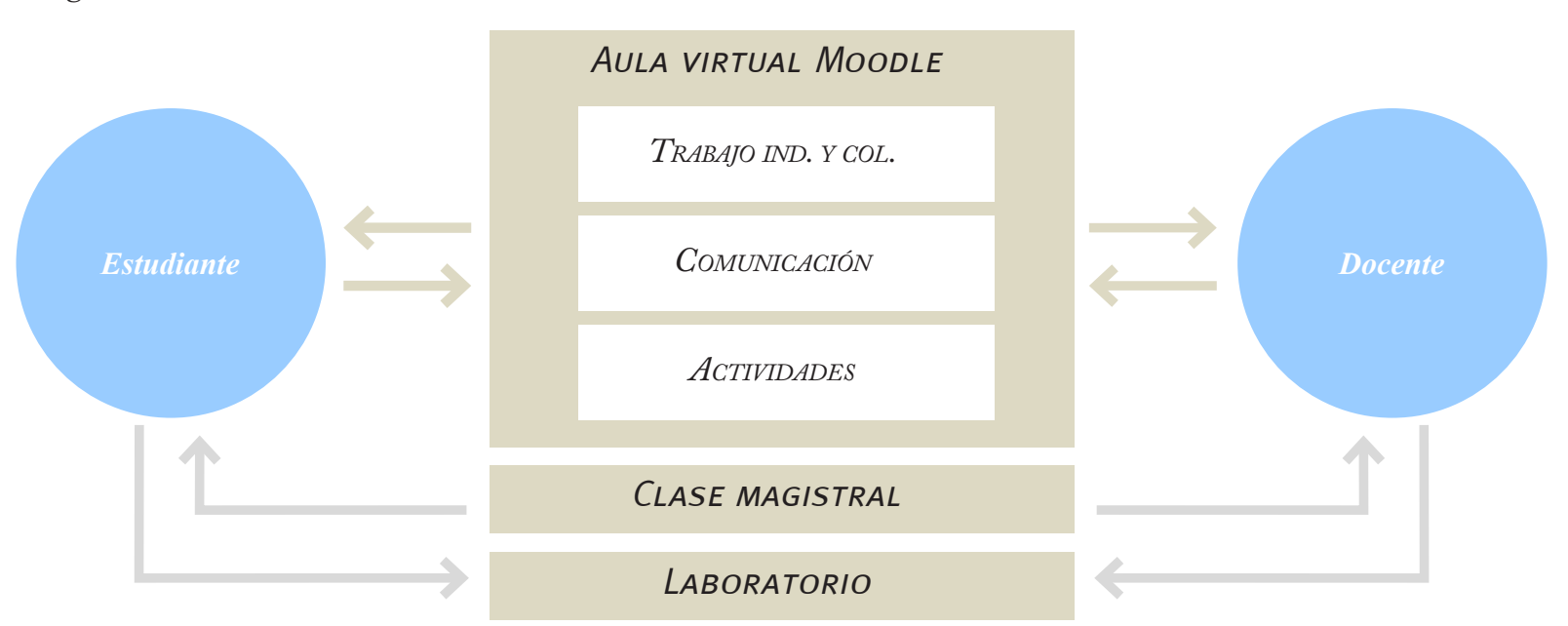

FIGURA 1. MAPA CONCEPTUAL DE LA METODOLOGÍA DE TRABAJO ACADÉMICO PROPUESTO

Estudiante. El alumno, dentro del esquema planteado, debe desarrollar en el curso unas horas de trabajo autónomo (HTA), las cuales son el fundamento de la formación y del aprendizaje. Este trabajo se desarrolla por medio del trabajo personal y del trabajo grupal.

El trabajo personal (HTA) es la fuente básica del aprendizaje y de la formación, e implica responsabilidades específicas del estudiante con respecto al estudio de las unidades temáticas; el trabajo grupal colaborativo (HTA) tiene como propósito el aprendizaje por medio de un equipo de trabajo, que implica la socialización de los resultados del trabajo personal, de actividades en equipo y elaboración de informes.

Docente. Dentro de este esquema, debe desarrollar horas de trabajo cooperativo (HTC) y horas de trabajo directo (HTD) por medio de: tutoría individual, tutoría en pequeños grupos y clase magistral.

La tutoría individual (HTC) es el acompañamiento que el docente hace al estudiante en horas lectivas de clase con carácter de asesoría al aprendizaje de los contenidos temáticos, evaluación de las actividades y seguimiento de su proceso formativo y de aprendizaje. La tutoría en pequeños grupos colaborativos (HTC) es el acompañamiento que el docente hace al estudiante en horas lectivas de clase a las actividades desarrolladas en pequeños grupos, revisión de informes, evaluación de las actividades y seguimiento de su proceso formativo y de aprendizaje. Por último, la clase magistral (HTD) son horas en las que el estudiante debe asistir de manera presencial; en este espacio el docente ejerce acompañamiento al conjunto de estudiantes a cargo, analiza la información relevante, sistematizada, y profundiza los conceptos claves del curso, es decir, expone los tópicos esenciales de los procesos de manufactura y desecha la información superflua.

\section{ASPECTOS DE METODOLOGĹA Y EVALUACIÓN}

Las características generales de las fases de aprendizaje del desarrollo del curso con apoyo virtual se establecen basadas en los lineamientos de la Universidad Nacional Abierta y a Distancia (UNAD), mediante las cuales se estructuran los dispositivos pedagógicos y didácticos de los cursos académicos en el contexto de la educación a distancia de la siguiente manera: 
Reconocimiento. Diseño de actividades que le permitan al estudiante pasar de las prenociones a las nociones. Es decir, motivarlo para que se involucre en los procesos iniciales de aprendizaje y active sus estructuras cognitivas. Consta de la revisión de presaberes.

Karolina

González

Guerrero |

Leonardo

Emiro Contreras

Bravo |

Alidis Cristina

Contreras Bravo |

Profundización. Diseño de situaciones y actividades de manera didáctica, conducentes a la apropiación de conceptos, teorías y procedimientos, según los propósitos, objetivos y competencias establecidos en el curso. En cada una de las unidades se desarrolla por medio de las lecciones evaluativas, quices en línea y presenciales, y parciales.

Transferencia. Diseño de actividades que deben agregar valores de recontextualización y productividad al conocimiento que se aprende y a las competencias derivadas (Padilla et al., 2008). La transferencia se promueve a través del trabajo grupal colaborativo y presencial. En la tabla 2 se muestran las actividades del curso (presencial-virtual), donde se pueden apreciar las actividades en línea (e-learning) y las actividades presenciales.

\section{TABLA 2.ACTIVIDADES DEL CURSO DE PROCESOS MECÁNICOS}

Etapa Actividad Forma de Evaluación

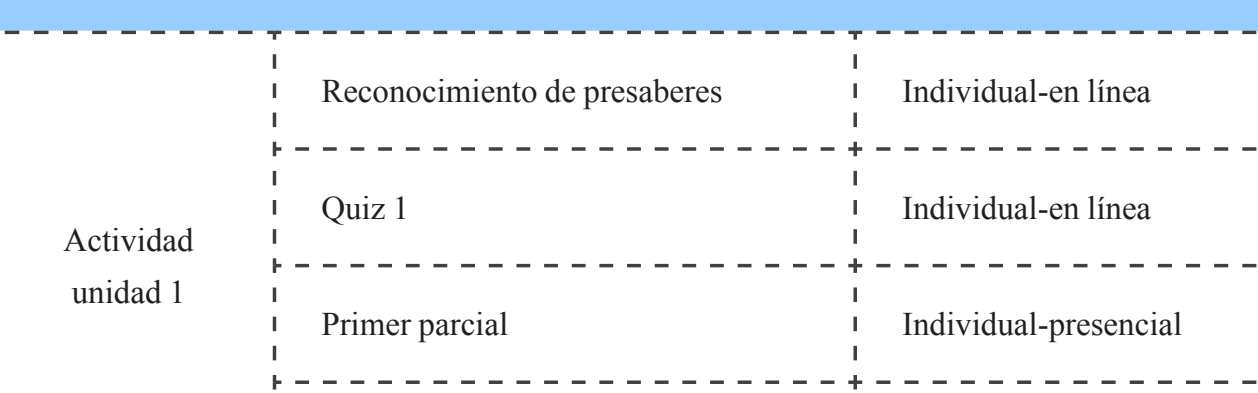

Trabajo grupal Col. $1 \mathrm{CAD} / \mathrm{CAM}$

Quiz 2

Actividad

unidad 2

Segundo parcial

Trabajo grupal Col. 2 CAD/CAM

Quiz 3

Actividad

unidad 3

Volumen 7

Número 13
Prueba final

Examen final
Fase de

Aprendizaje

Reconocimiento

Profundización

Profundización

Transferencia

Profundización

Profundización

Transferencia

En línea-presencial

Individual-en línea

Profundización

Transferencia

Individual-presencial

Profundización

Individual-presencial

En línea-presencial

Transferencia

Individual-presencial

Profundización

a


A continuación se explican brevemente cada uno de los elementos y actividades que se desarrollan en el aula virtual de procesos mecánicos (v. tabla 3):

\section{TABLA 3. ACTIVIDADES DEL CURSO VIRTUAL}

\section{Elementos del curso virtual}

\section{Tópico cero}

Noticias del curso (foro): Es una actividad de Moodle tipo FORO. Permite sostener conversaciones con el docente por medio tradicional o a través de este foro.

Agenda del curso (página web): Es la agenda de actividades del período académico, contiene información como: la actividad, una breve descripción, entre otras.

\section{Tópico uno}

Revisión de presaberes (lección evaluativa): Busca generar el interés en el estudiante al iniciar la asignatura. Se lleva a cabo por medio de una lección evaluativa en la que se revisan sus estudios anteriores, así como los elementos en sus conocimientos que fundamentarán el inicio y desarrollo del curso. Esta se lleva a cabo por medio de tres lecturas y de preguntas relacionadas con las lecturas.

Software CAD/CAM (base para trabajo colaborativo): Esta sección posee información acerca del proceso de descarga e instalación del software; así como lecciones de aprendizaje sobre este.

\section{Tópico dos: actividades unidades}

Contenido (.pdf o .avi): Comprende información que se presentan en formato pdf y videos de los procesos de fabricación o de simulaciones de los procesos.

Trabajo colaborativo unidad (foro y tarea): Son grupos de trabajo establecidos por estudiantes. Aunque para el desarrollo de los trabajos grupales existen muchas estrategias de aprendizaje definidas por distintos autores, las cuales pueden ser implementadas, en los foros se usan: aprendizaje basado en proyectos, aprendizaje basado en problemas (APB) y aprendizaje colaborativo.

Quiz unidad (quiz): Estos son cortos (entre 15 y 20 minutos) y se diseñan con preguntas tipo I, II, III y IV del sistema Ecaes, para entrenar a los estudiantes en este tipo de pruebas. Cada una de las unidades tendrá estos mismos elementos.

- - - - - - - - - - - - - - - - - - - - - - - - - - - - - - - - - - - - - - - - -

Algunas de las actividades propuestas se explicaran brevemente con el fin de ejemplificar el uso por parte del estudiante y por parte del docente. La figura 2 muestra como es el inicio del curso de Procesos Mecánicos en línea. 


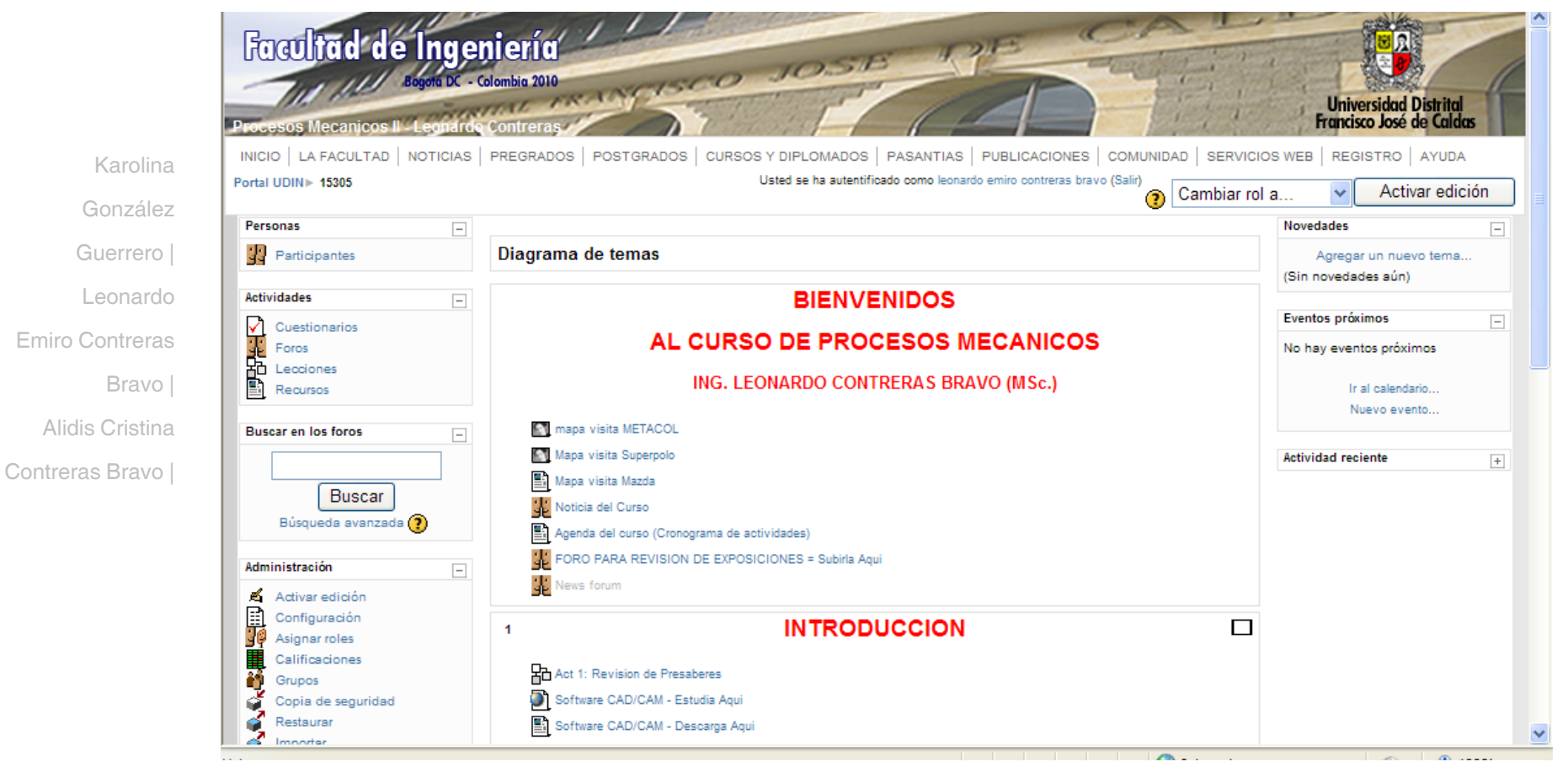

FIGURA 2. PANORAMA GENERAL DEL CURSO VIRTUAL

Agenda del curso: Contiene información como: la unidad de estudio, la actividad, fecha de entrega, información de si la actividad se realiza en línea o de manera tradicional y del valor (\%) de la actividad realizada (v. figura 3).

Software CAD/CAM: Contiene información acerca de cómo descargar e instalar el software CAD (diseño asistido por computador) / CAM (manufactura asistida por computador) denominado SpectraCAM Turning; asimismo contiene lecciones de aprendizaje del mismo software (v. figura 4), para que el estudiante pueda descargarlo y con esto realizar su lectura en línea y fuera de línea (Contreras y Vargas, 2010). 


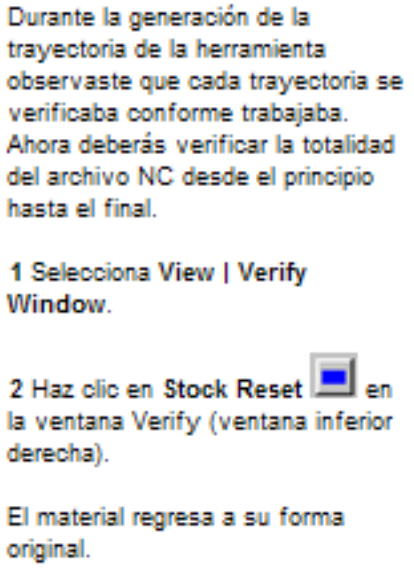

\section{Tarea: Verificación del archivo NC}

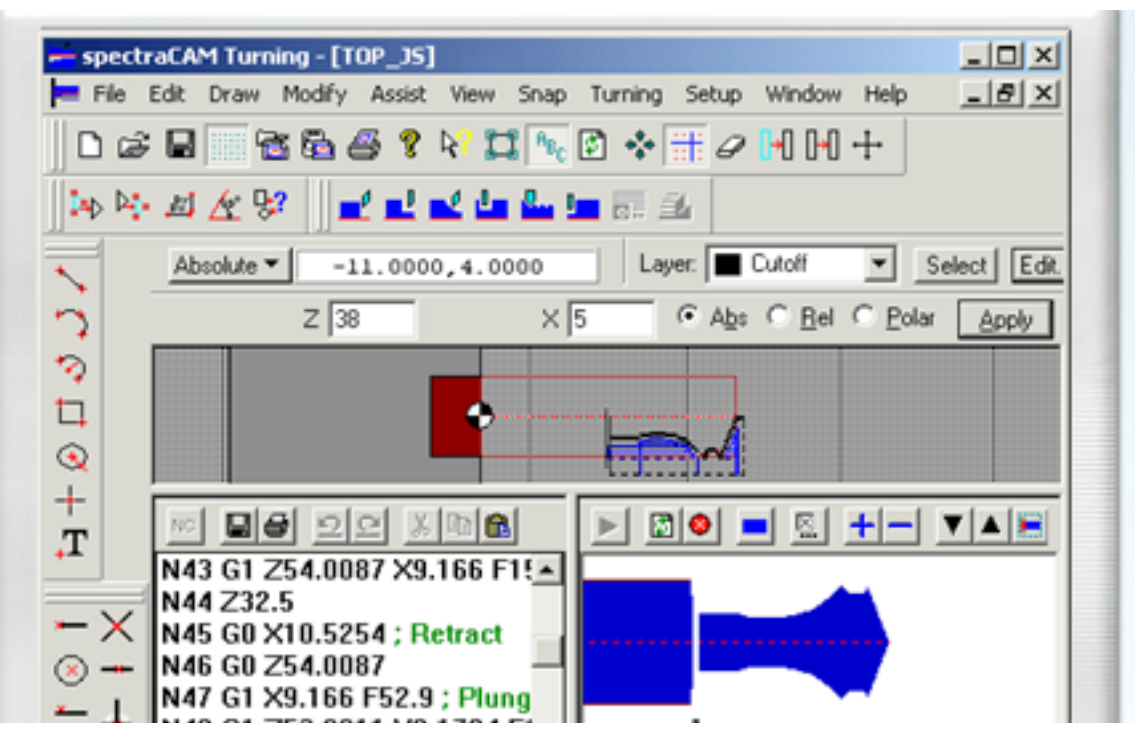

Trabajo grupal colaborativo CAD/CAM: Son diversas actividades programadas y orientadas a que todos los miembros del grupo logren un aprendizaje significativo debido a los diversos aportes de cada uno al referirse a un problema de su campo laboral. Esta actividad posee guías con las especificaciones del trabajo, productos esperados y rúbrica de evaluación (Gómez et al., 1996).

Parciales, examen final, exposición, laboratorio: Cada una de estas actividades está relacionada con un curso de procesos en la enseñanza tradicional. El laboratorio constituye una práctica de mecanizado, procesos de soldadura y de fundición.

El instrumento de recopilación de datos se aplicó a 64 alumnos pertenecientes a la asignatura Procesos Mecánicos de los semestres 2010-I, 2010-II. El instrumento de recopilación de datos incluyó los siguientes ítems y preguntas de la tabla 4.

\section{TABLA 4. PREGUNTAS DEL INSTRUMENTO}

¿Desde dónde accede al curso virtual?
Sistemas
de accesso a
aula web




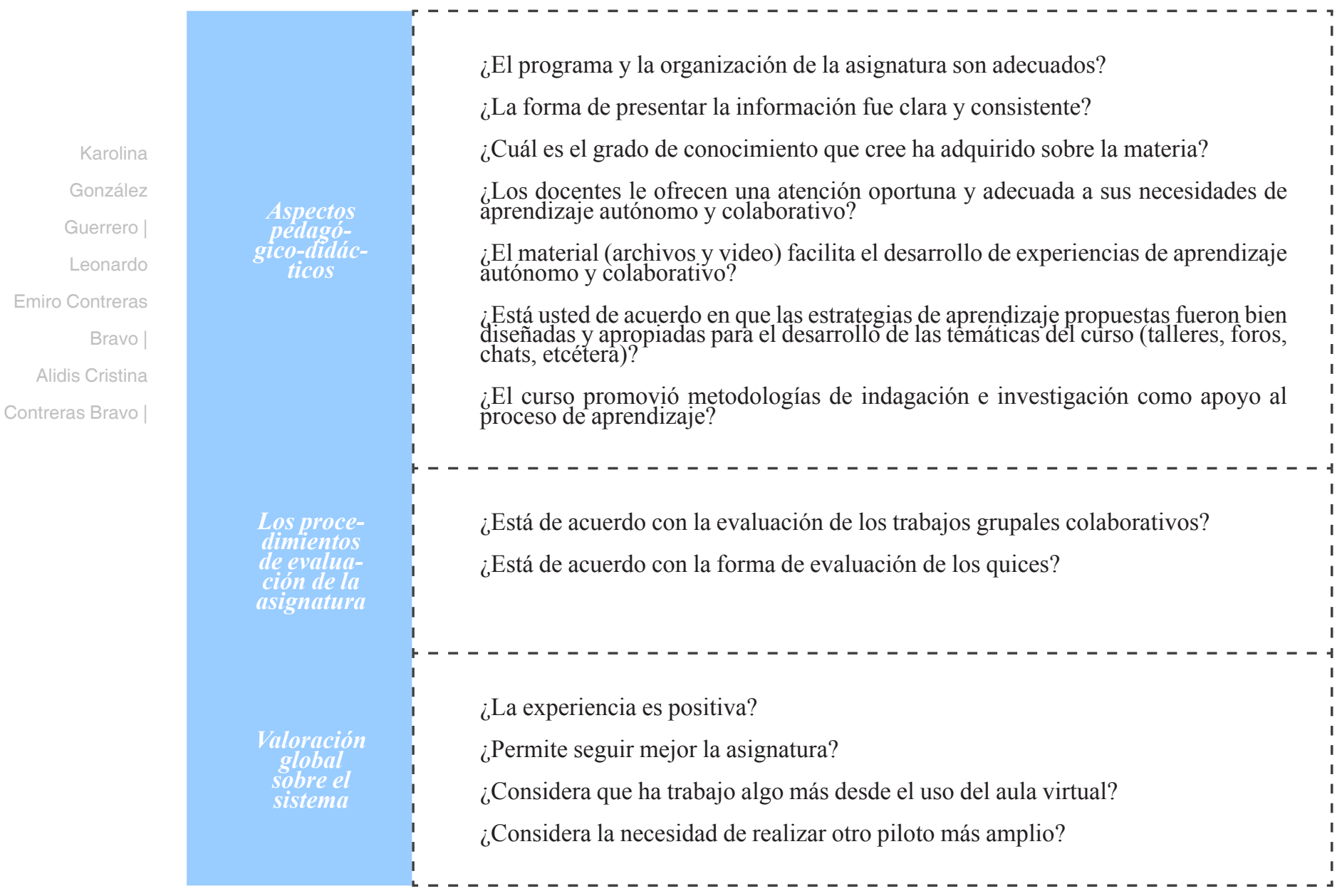

\section{RESULTADOS Y DISCUSIÓN}

Las calificaciones promedio obtenidas mediante el sistema presencial-tradicional $(31,1)$ son un poco inferiores a las obtenidas por los estudiantes mediante la aplicación de la enseñanza b-learning $(34,5)$, como se muestra en la figura 5. En cuanto a la desviación estándar, el grupo que recibió enseñanza tradicional tuvo un valor de 5,49, mientras que el de enseñanza b-learning tuvo un valor de 4,23. En este caso se puede concluir que la aplicación de la metodología dio buenos resultados, aunque se espera que en una segunda etapa de aplicación (extensión a demás asignaturas relacionadas) los resultados sean mejores.

Con respecto a "la facilidad de usar la plataforma Moodle y los servicios que la integran", el 97\% ha manifestado estar de acuerdo en que es fácil de usar en diferentes grados. La lectura de estos resultados ratifica que el recurso tecnológico curso virtual en línea y plataforma Moodle no presenta mayores dificultades en su utilización, incluso para alumnos que tienen debilidades en cuando al conocimiento y el acceso a los recursos informáticos.

Panorama | pp. $11-23$ | Volumen 7 | Número 13
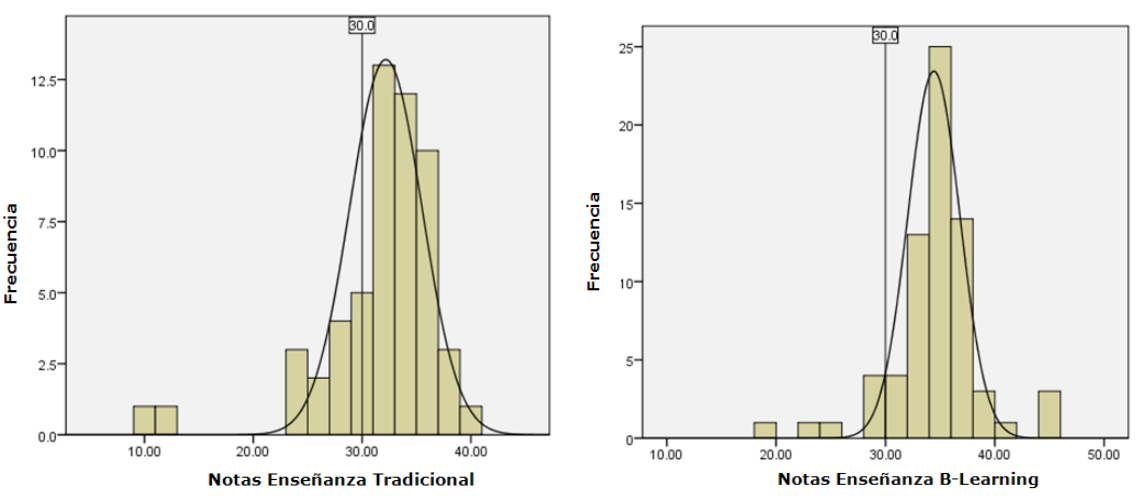
Se pudo observar la buena calificación obtenida para la enseñanza del software CAD/CAM mediante el curso virtual creado en Moodle. Es destacable el 48\% y 36\% para las respuestas "bastante" y "totalmente" respecto a la pregunta “¿La enseñanza del software CAD/CAM por medio del curso virtual le pareció acertada?”.

En relación con si “¿El programa y la organización de la asignatura son adecuados?”, un 45\% manifestó que era "bastante" adecuado, y un 42\% manifestó que era "totalmente" adecuado. Esto da pie para decir que el trabajo realizado con anterioridad para identificar aspectos relacionados con el contenido de la asignatura, en el que se realizó un paralelo entre el pensum y los contenidos de Ingeniería Industrial de la Universidad Distrital con otras universidades públicas y privadas de índole nacional e internacional, fue adecuado.

En relación con la pregunta “¿Está usted de acuerdo en que las estrategias de aprendizaje propuestas fueron bien diseñadas y apropiadas para el desarrollo de las temáticas del curso?”, cabe resaltar que un 65\% considera que fueron "bastante" acertadas. Este apartado se dedicó a averiguar estrategias para contrarrestar el problema del estudiante para ser activo en su aprendizaje, motivado por los modelos tradicionales de enseñanza y, sobre todo, porque no aprecia la utilidad de este aprendizaje para el rendimiento en los exámenes, pues normalmente estos premian el aprendizaje mecánico o memorístico.

En relación con la forma como se evalúan los trabajos grupales colaborativos, un 61\% manifestó que estaba "totalmente" de acuerdo, y un 36\% manifestó que estaba "bastante" de acuerdo. Las respuestas sobre la forma de evaluación de los quices también fueron satisfactorias, ya que un 45\% manifestó que estaba "bastante" de acuerdo y un 36\% manifestó que estaba "totalmente" de acuerdo. Aunque las respuestas parecen ser buenas, también hay ciertos aspectos por mejorar, como el banco de preguntas de los quices, el cual debe ir aumentando con el pasar de los semestres.

Entre las ventajas planteadas por los estudiantes se pueden destacar las siguientes: disponibilidad y planeación de tiempo del alumno y del docente; permite consultar y descargar material de estudio 24 horas al día; y permite adquirir conocimientos de software a los que no se puede acceder en clase por falta de tiempo, espacio y equipos. La principal desventaja es la dificultad del acceso a la plataforma, sobre todo cuando lo hacen desde fuera de la facultad (según la encuesta no hubo inconvenientes de este estilo).

\section{Conclusiones}

Las nuevas tecnologías no solo inducen ciertas habilidades y capacidades en el estudiante, sino que permiten superar algunas de las limitaciones presentes en la enseñanza tradicional, como los límites de espacio y tiempo, ya que es posible dar al estudiante cursos de formación por medio de internet o intranet, los cuales en cierta medida permitirían disminuir los costos de personal docente, del aula equipada, de mantenimiento de equipos y software.

Aunque es importante el uso de las tecnologías de información en el aula de clase, cabe mencionar que una herramienta informática no podrá suplir totalmente la relación docente alumno; tal como lo manifiestan González y León (2010):

\section{El CAD no es sino una forma de plasmar los conocimientos que adquirimos mediante la enseñanza tradicional, y que de ningún modo podría sustituir a la Expresión Gráfica, porque el estudiante debe manejarse en los distintos sistemas de representación más usuales en ingeniería, garantizando un minimo de visión espacial y de destreza operativa realizando sus ejercicios mediante las herramientas clásicas. (Gómez, et al., 1996)}

Los riesgos que se corren con el trabajo grupal colaborativo (modelo constructivista) pueden ser: primero, que la formación de grupos no garantiza que los estudiantes trabajen colaborativamente, ya que se puede pensar que es mejor metodología

b-learning en la enseñanza de procesos 
o más fácil repartirse el trabajo para después juntar las partes de cada quien en un reporte final (enseñanza tradicional). Segundo, que, como sucede tanto en la educación a distancia como en la presencial, en los equipos se presentan pseudoalumnos que tratan de beneficiarse del trabajo de sus compañeros, sin que ellos hagan el esfuerzo necesario.

Karolina

González

Guerrero |

Leonardo

Emiro Contreras

Bravo |

Alidis Cristina

Contreras Bravo |

La incursión del b-learning fue una metodología que proporcionó experiencias educativas de calidad en formatos innovadores (internet e informática), los cuales estimularon la participación e integración en espacios de comunicación y la colaboración entre los actores del proceso educativo. Asimismo, esta metodología constituye un primer paso para la disminución de las resistencias al cambio no solo de docentes y estudiantes, sino también de directivos.

Este trabajo fue un piloto de la incursión de TIC en el programa de Ingeniería Industrial de la Universidad Distrital. En este momento, en el curso de Procesos en particular, se han implementado una serie de herramientas tecnológicas (software, aplicativos, etcétera) con el fin de ayudar a la mejora del aprendizaje de los procesos industriales usados en el país. En cuanto al área de diseño y manufactura se han desarrollado herramientas para el desarrollo de las habilidades espaciales de los estudiantes de Ingeniería, con lo cual se espera, luego de casi dos años de implementación, obtener resultados favorables para el desarrollo de estas capacidades. 
1. Andrade, L. (2007). Ambientes de aprendizaje para la educación en tecnología. Bogotá, Colombia: Departamento de Tecnología, Universidad Pedagógica Nacional.

2. Arias, N.; González, K. y Padilla,J. (2010). Educación a distancia y educación virtual: una diferencia necesaria desde la perspectiva pedagógica y la formación de ser humano. Revista UNAD, 9(3), $207-221$.

3. Contreras, L. E. y Vargas, L. F. (2010). Consideraciones sobre la tecnología educativa en la enseñanza del diseño y la manufactura en ingeniería industrial. Dialectica, 27, 9-15, ISSN 0123-2592.

4. García, P. y Lacleta, M. (2006). Moodle: difusión y funcionalidades. Recuperado de: http://www.unizar. es/eees/ innovacion06

5. Garrido, M. (2005). El reto del cambio educativo: nuevos escenarios y modalidades de formación. Recuperado de: http://tecnologiaedu.us.es/formaytrabajo/Documentos/lin7fan.pdf.

6. Gómez, E. et al. (1996). Dibujo tradicional y dibujo asistido por ordenador, ¿incompatibles o complementarios?. En Actas del VIII Congreso Internacional de Ingenieria Gráfica (pp. 109-121), Jaen, España: Universidad de Jaen.

7. González, K. y León, J. C. (2010). Incorporación gradual de tecnologías de la información y la comunicación en programas de educación superior. Dialéctica, 27, 137-143, ISSN 0123-2592.

8. González, K.; Padilla, J. y Arias, N. (2010). Incidencia del estilo de aprendizaje en el rendimiento académico en un curso virtual. Revista virtual Universidad Católica del Norte, 31, 6-24, ISSN 0124-5821.

9. Padilla, J.et al. (2008). Creencias de los docentes acerca del uso de las tecnologías de la información y comunicación. Revista Educación y Desarrollo Social, 2 (2), 45-57.

10. Rodríguez, G.; Gil, J. y García, E. (1999). Metodología de la investigación cualitativa. Málaga, España: Aljibe.

11. Rogoff, B. (1998). Aprendices del pensamiento. El desarrollo cognitivo en el contexto social. Barcelona, España: Paidós.

12. Sandoval, C. (2002). Módulo 4. Investigación cualitativa. Medellín, Colombia: INER- Universidad de Antioquia. 